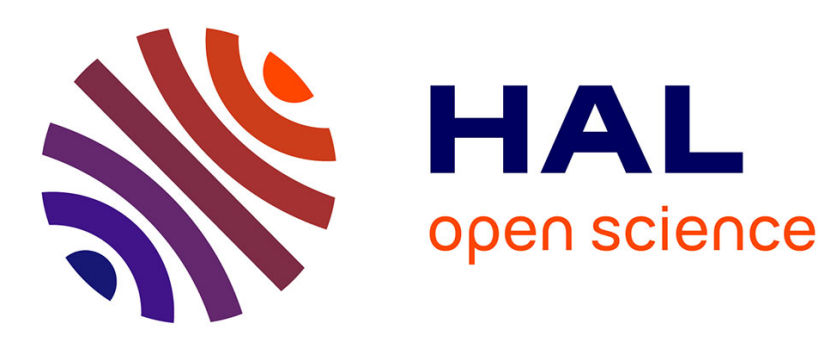

\title{
Forces and the existence of stresses in invariant continuum mechanics \\ Reuven Segev
}

\section{To cite this version:}

Reuven Segev. Forces and the existence of stresses in invariant continuum mechanics. Journal of Mathematical Physics, 1986, 27 (163), 8 p. hal-00957447

\section{HAL Id: hal-00957447 \\ https://hal.science/hal-00957447}

Submitted on 10 Mar 2014

HAL is a multi-disciplinary open access archive for the deposit and dissemination of scientific research documents, whether they are published or not. The documents may come from teaching and research institutions in France or abroad, or from public or private research centers.
L'archive ouverte pluridisciplinaire HAL, est destinée au dépôt et à la diffusion de documents scientifiques de niveau recherche, publiés ou non, émanant des établissements d'enseignement et de recherche français ou étrangers, des laboratoires publics ou privés. 


\title{
Forces and the existence of stresses in invariant continuum mechanics
}

\author{
Reuven Segev \\ Department of Mechanical Engineering, Pearlstone Center for Aeronautical Engineering Studies, Ben Gurion \\ University, Beer Sheva, Israel
}

\begin{abstract}
In an invariant formulation of $p$ th-grade continuum mechanics, forces are defined as elements of the cotangent bundle of the Banach manifold of $C^{p}$ embeddings of the body in space. It is shown that forces can be represented by measures which generalize the stresses of continuum mechanics. The mathematical representation procedure makes the restriction of forces to subbodies possible. The local properties of the stress measures are examined. For the case where stresses are given in terms of smooth densities, it is shown that the structure of forces agrees with the form of forces one assumes in the traditional formulation, and the equilibrium differential equations are obtained.
\end{abstract}

\section{INTRODUCTION}

It is well known that the laws of continuum mechanics, the mechanics of deformable bodies, cannot be deduced from the laws of mechanics of material points and rigid bodies. Additional assumptions are introduced and new notions such as internal forces, external forces, stresses, and the equilibrium equation emerge.

The geometric framework in which the classical theory of continuum mechanics is developed is the three-dimensional Euclidean space. The following paragraphs review the basic structure of the theory.

The first basic assumption made in continuum mechanics regarding the nature of forces is that the total force acting on a body $B$ is of the form

$$
f=\int_{B} b d v+\int_{\partial B} t d a,
$$

where $b$ is a continuous vector field, called the body force, defined in the body, and $t$ is a continuous vector field, called the surface force, defined on the boundary of the body. The basic problem of continuum mechanics is encountered when we try to restrict a given force on $B$ to a subbody. Considering a subbody $P$ of the body $B$, the total force $f_{P}$ acting on it should also be given in terms of a body force and a surface force as in Eq. (1). In general, the fields $b$ and $t$ associated with the subbody $P$ are different from those given on $B$. In particular, physical experience shows that even if $P$ is disjoint from the boundary of $B$, a surface force acts on the boundary of $P$. This newly emerged surface force is traditionally termed internal force or traction as it may be interpreted as the force that is applied on $P$ by its complement in the body. Thus, the values of $b$ and $t$ at a point $X \in P$ will depend in general on the subbody $P$ under consideration and we write

$$
b=b(X, P), \quad t=t(X, P) .
$$

The next assumption, called Cauchy's postulate, deals with the dependence of $b$ and $t$ on $P$. It states that $b$ does not depend on $P$ so that $b=b(X)$, and that the surface force depends on $P$ only through the unit vector $n$ perpendicular to the boundary of $P$ at $X$, i.e., $t=t(X, n)$. Clearly, this last hypothesis does not provide all the necessary information needed in order to determine $t$.
Assuming that the total force on each subbody of the body $B$ vanishes, it is possible to prove the following results.

There exists a tensor field $\sigma$ in the body such that

$$
t(X, n)=\sigma(X)(n(X)) \text {. }
$$

The tensor field $\sigma$ is the stress field, and it has to satisfy the differential equation

$$
\operatorname{div} \sigma+b=0 \text { in } B \text {. }
$$

If we assume in addition that the total moment on each subbody of $B$ vanishes, we find that $\sigma$ is symmetric.

From Eqs. (1) and (2) it is clear that if $\sigma$ is given, one can associate a unique body force field and a unique surface force field with each subbody. However, the differential equation (3), known as the equlibrium equation, and the boundary condition (2) cannot determine the stress uniquely for given $b$ and $t$ on $B$. This lack of uniqueness in the determination of the stress field means that the force on a body cannot be restricted to subbodies in a unique fashion. In order to determine the stress field, constitutive relations are introduced. The constitutive relations, obtained by physical experiments, relate the stress with the configuration of the body and supply all the necessary information so that the stress can be determined uniquely. Clearly, using Eq. (2), $\sigma$ can be determined uniquely if $t$ is given for every subbody $P$ of $B$.

Modern attempts to axiomatize the theory of forces and stresses can be found in Gurtin and Williams, ${ }^{1}$ Gurtin and Martins, ${ }^{2}$ and Truesdell. ${ }^{3}$ The authors postulate a system of axioms describing the properties of forces in general. In addition, they assume equilibrium and they assume that external forces are composed of body forces that are absolutely continuous with respect to the volume measure, and surface forces that are absolutely continuous with respect to the surface area of the body. With these assumptions the authors prove that forces are given in the form of Eq. (1) and that Cauchy's postulate holds. Marsden and Hughes ${ }^{4}$ have generalized the theory to Riemannian manifolds using an invariance principle for an assumed form of a balance of energy where they assume the transformation rules for the various variables including $b$ and $t$.

During the 1960's, in an attempt to formulate theories that would account for interactions that are more complicated than those afforded by the classical theory, the theories of couple stresses and the theories of materials of grade $p>1$ 
were developed. A historical account of the subject together with a review of the various approaches can be found in Truesdell and $\mathrm{Noll}^{5}$ (pp. 389-401). For a variety of applications one can consult Mindlin ${ }^{6}$ and references cited therein. Unlike Cauchy's theory, the theories of materials of grade $p$ are based on energy principles in which the potential energy density is assumed to depend on derivatives of order $p$ of the deformation.

In this paper we propose a theory of forces and stresses based on the principle that forces should be defined as elements of the cotangent bundle $T^{*} Q$ of and appropriate configuration manifold $Q$. Specifically, we show that $p$ th-order continuum mechanics corresponds to the case where the configuration space is the set of all $p$-times differentiable embeddings of the body in space equipped with the $C^{p}$ topology. It turns out that in this case forces can be represented by measures on the pth jet bundle over the body where the representing measures generalize the stresses. For example, it follows that in first-order continuum mechanics and the case of three-dimensional Euclidean geometry [where the first jet bundle can be identified with $\left.B \times R^{3} \oplus L\left(R^{3}, R^{3}\right)\right]$ any force can be represented in the form

$$
f(u)=\int_{B} u^{i} d \mu_{i}+\int_{B} u_{, j}^{i} d \mu^{j}{ }_{i},
$$

where $\mu_{i}$, and $\mu^{j}{ }_{i}$ are the components of a measure over $B$ valued in $R^{3} \oplus L\left(R^{3}, R^{3}\right)$. The first three components vanish if a Euclidean symmetry requirement is imposed and the $\mu^{j}{ }_{i}$ correspond to the stress. If these measures are differentiable with respect to the volume measure, their densities are the components of the stress field. In the more general case where a connection is specified on the space manifold, any force can be represented in the form

$$
f(u)=\sum_{k=0}^{p} \int_{B} \nabla^{k} u d \sigma_{k}
$$

where $\nabla^{k}$ is the $k$ th covariant derivative and the $\left\{\sigma_{k}\right\}$ are the representing measures.

The resulting structure has the following features.

(a) The theory applies in the general geometry of differentiable manifolds.

(b) The definition of a force extends the definition given in the case of finite-dimensional classical mechanics by Arnold $^{7}$ and Tulczyjew ${ }^{8}$ to the infinite-dimensional case. Thus, it clarifies the point of departure of continuum mechanics from analytical mechanics.

(c) Some assumptions made in the classical construction, such as the form (1) of the forces on bodies, are obtained mathematically as results of the definition of forces.

(d) The theory links the properties of forces and stresses with the axiom of impenetrability.

(e) The theory allows stresses which are as irregular as measures, while the classical theory deals with continuous stresses only.

(f) The theories of materials of grade $p$ are generalized to differentiable manifolds. The suggested formulation is free of any energy considerations and the relation between the theory of materials of grade one and materials of a higher grade is clear and simple. The grade of a material is a conse- quence of the choice of a topology on the set of configurations.

(g) A generalized form of the equilibrium equation is obtained as a result of the mathematical procedure, and the origin of the nonuniqueness in the relation between stresses and forces is explained.

(h) A simple constitutive theory is suggested in which body self-determinism and continuity imply jet locality.

\section{THE BASIC STRUCTURE}

Definition 2.1: A body is a compact differentiable manifold with corners. A typical body will be denoted by $B$ and its dimension will be denoted by $m$.

Definition 2.2: The physical space is a differentiable manifold $S$ without a boundary.

Definition 2.3: A configuration of class $p$ is a $C^{P}$ embedding of a body $B$ in the physical space for $p>1$.

The requirement that a configuration of a body into space is an embedding is a result of two traditional principles: the principle of impenetrability stating that one portion of the matter never penetrates within another, and the principle of permanence of matter stating that no region of positive finite volume is deformed into one of zero or infinite volume (cf. Truesdell and Toupin, ${ }^{9}$ pp. 234-244).

For a fixed body $B$ and a given $p$, the configuration space $Q$ is the set of all configurations of class $p$ of the body in space.

We recall ${ }^{10-13}$ that the set $C^{p}(B, S)$ of $C^{p}$ mappings of $B$ into $S$ can be given the structure of a Banach manifold. For any $\kappa \in C^{p}(B, S)$, the tangent space $T C^{p}(B, S)_{\kappa}$ can be identified with $C^{p}\left(\kappa^{*} \tau_{S}\right)$, the Banachable space of $C^{p}$ sections of the pullback of the tangent bundle $\tau_{S}$ by $\kappa$. The Banach space topology of $C^{p}\left(\kappa^{*} \tau_{S}\right)$ is given as follows. Let $v: K \rightarrow R^{n}$ be a $C^{P}$ mapping defined on a compact set $K$. We use the notation

$$
\|v\|_{p}=\max _{j} \sup _{x \in K}\left\{\left|D^{j} v(x)\right|\right\}, \quad 0 \leqslant j \leqslant p .
$$

Clearly, \|\|$_{p}$ is a norm for the space of all such $C^{p}$ mappings.

Now, let $B_{1}, \ldots, B_{r}$ be a covering of $B$ by compact submanifolds of the same dimension as $B$ such that each $B_{i}$ is contained in the domain of a vector bundle chart $\psi_{i}$ of $\kappa^{*} \tau_{S}$. Then for $u \in C^{p}\left(\kappa^{*} \tau_{S}\right)$ define

$$
\|u\|=\max _{i}\left\|\underline{u}_{i}\right\|, \quad i=1, \ldots, r,
$$

where $\underline{u}_{i}$ is the local representative of $u$ in the chart $\psi_{i}$. Again, \|\| is a norm on $C^{p}\left(\kappa^{*} \tau_{S}\right)$ and any other norm induced by another covering will induce an equivalent topology on $C^{p}\left(\kappa^{*} \tau_{s}\right)$.

The tangent space $T C^{P}(B, S)_{\kappa}$ can also be identified with the vector space of vector fields along $\kappa$, i.e., $\left\{u \in C^{P}(B, T S) ; \quad \tau_{S}{ }^{\circ} u=\kappa\right\}$.

In addition, we recall that since $p>1$, the set of $C^{p} \mathrm{em}$ beddings is an open subset ${ }^{14}$ of $C^{P}(B, S)$. Hence, $Q$ is a Banach manifold and we have 
$T Q_{\kappa} \cong C^{p}\left(\kappa^{*} \tau_{S}\right) \cong\left\{u \in C^{p}(B, T S) ; \quad \tau_{S}{ }^{\circ} u=\kappa\right\}$.

An element of $T Q$ is a virtual displacement, a term motivated by the second interpretation we gave of $T Q_{\kappa}$.

Definition 2.4: A force (of grade $p$ ) is an element of the cotangent bundle $T^{*} Q$.

Let $f \in T^{*} Q_{\kappa}$ and $u \in T Q_{\kappa}$ for some configuration $\kappa$. The evaluation $f(u)$ is traditionally called the virtual work performed by the force $f$ on the virtual displacment $u$.

The basic structure, as defined in this section, has been given for the finite-dimensional configuration space by Arnold ${ }^{7}$ and Tulczyjew. ${ }^{8}$ In the infinite-dimensional case considered here, the specification of the class of admissible configurations and the topology chosen will determine the nature of forces. It is our aim to study the consequences of these choices and to show that the basic properties of forces and stresses in continuum mechanics can be obtained naturally in the suggested framework.

\section{THE REPRESENTATION OF FORCES BY STRESSES AND THE PRINCIPLE OF VIRTUAL WORK}

Given $\kappa \in Q$, the identification of $T Q_{\kappa}$ with $C^{p}\left(\kappa^{*} \tau_{\mathrm{S}}\right)$ allows us to identify the forces in $T^{*} Q_{\kappa}$ with section distributions in $C^{p}\left(\kappa^{*} \tau_{S}\right)^{*}$. Thus, the problem of restriction of forces from a given body to its subbodies means mathematically that we have to study the restrictions of $C^{p}$ section distributions.

Consider the jet extension mapping

$$
j_{p}: \quad C^{p}\left(\kappa^{*} \tau_{S}\right) \rightarrow C^{0}\left(J^{p}\left(\kappa^{*} \tau_{s}\right)\right) .
$$

We note that $j_{p}$ is linear, injective and if we use natural charts on both $\kappa^{*} \tau_{\mathrm{S}}$ and $J^{p}\left(\kappa^{*} \tau_{S}\right)$ and norms induced on $C^{p}\left(\kappa^{*} \tau_{S}\right)$ and $C^{0}\left(J^{p}\left(\kappa^{*} \tau_{S}\right)\right)$ by these charts, we observe that $j_{p}$ is also norm preserving. It follows that every force in $C^{p}\left(\kappa^{*} \tau_{S}\right)^{*}$ is of the form $j_{p}^{*}(\sigma)$ for some $\sigma \in C^{0}\left(J^{p}\left(\kappa^{*} \tau_{S}\right)\right)^{*}$, where

$$
j_{p}^{*}: \quad C^{0}\left(J^{p}\left(\kappa^{*} \tau_{S}\right)\right)^{*} \rightarrow C^{p}\left(\kappa^{*} \tau_{S}\right)^{*}
$$

is the adjoint of the jet extension mapping. The elements of $C^{0}\left(J^{p}\left(\kappa^{*} \tau_{s}\right)\right)^{*}$ are called stresses. Hence, if $f=j_{p}^{*}(\sigma)$, we have $f(u)=\sigma\left(j_{p}(u)\right)$ for every virtual displacement $u$ and we say that the stress $\sigma$ represents the force $f$. This is a generalization of the principle of virtual work in continuum mechanics which states that the virtual work performed by the force on a virtual displacment is equal to the virtual work performed by the stress on the derivative of the virtual displacement.

\section{LOCAL PROPERTIES OF STRESSES}

By their definition, forces are special types of section distributions or currents (Choquet-Bruhat et al. ${ }^{15} \mathrm{pp} .400-$ 406, DeRham, ${ }^{16}$ Schwartz ${ }^{17}$ ) and stresses that belong to a simpler class of distributions (measures) represent them. In this section we consider the local properties of stresses.

Let $B_{1}, \ldots, B_{r}$ be compact submanifolds of $B$ of the same dimension as $B$ whose interiors cover $B$ and let $\phi_{1}, \ldots, \phi_{r}$ be a $C{ }^{\infty}$ partition of unity such that supp $\phi_{i}$ Cinterior $B_{i}$. It can be shown that if $\pi$ is a vector bundle over $B$, then

$$
\begin{array}{r}
\widetilde{C}^{0}(\pi)=\left\{\left(w_{1}, \ldots, w_{r}\right) \in \underset{i=1}{\oplus} C^{0}\left(\pi \mid B_{i}\right) ;\right. \\
\left.w_{i}\left|B_{i} \cap B_{j}=w_{j}\right| B_{i} \cap B_{j}\right\}
\end{array}
$$

is isomorphic to $C^{0}(\pi)$. The isomorphism is given by $w \mapsto\left(w\left|B_{1}, \ldots, w\right| B_{r}\right)$ and its inverse given by $\left(w_{1}, \ldots w_{r}\right) \mapsto \Sigma_{i} \tilde{w}_{i}$, where $\tilde{w}_{i} \in C^{0}(\pi)$ is given by $\tilde{w}_{i} \mid B_{i}=\phi_{i} w_{i}$ and $\tilde{w}_{i}=0$ outside $B_{i}$ (for a complete proof see Palais, ${ }^{10}$ pp. 10 and 11 ).

Given vector bundle charts

$$
\Psi_{i}: \pi \mid B_{i} \rightarrow K \times R^{N}, \quad K \subset R^{m}
$$

(assuming that $B$ is $m$-dimensional and that the fiber of $\pi$ is $N$-dimensional) and denoting by $\psi_{i}$ the first component of $\Psi_{i}, \quad C^{0}\left(\pi \mid B_{i}\right)$ can be identified with $C^{0}\left(\psi_{i}\left(B_{i}\right)\right)^{N}$, the space of $N$-tuples of continuous real valued functions on $\psi_{i}\left(B_{i}\right)$ for each $i$. Thus, $C^{0}(\pi)$ is isomorphic with ${ }^{10}$

$$
\begin{aligned}
& \left\{\left(w_{1}, \ldots, w_{r}\right) \in \underset{i=1}{\oplus} C^{0}\left(\psi_{i}\left(B_{i}\right)\right)^{N} ;\right. \\
& \left.\quad \Psi_{i}^{-1} \circ w_{i} \circ \psi_{i}=\Psi_{j}^{-1} \circ w_{j} \circ \psi_{j} \text { on } B_{i} \cap B_{j}\right\}
\end{aligned}
$$

via $w \mapsto\left(w_{i}, \ldots, w_{r}\right)$, where $w_{i}=\Psi_{i} \circ w \mid B_{i} \circ \psi_{i}^{-1}$, and the inverse is given by $w=\Sigma_{i} \tilde{w}_{i}$, where $\tilde{w}_{i} \in C^{0}(\pi)$ are given by $\tilde{w}_{i} \mid B_{i}=\phi_{i}\left(\Psi_{i}^{-1} \circ w_{i} \circ \psi_{i}\right)$ and $\tilde{w}_{i}=0$ outside $B_{i}$.

We conclude, therefore, that given a partition of unity, a vector bundle atlas, and $\mu \in C^{0}(\pi)^{*}$, there exists a collection $\left\{\mu_{\tau_{i}}\right\}, i=1, \ldots, r, \mu_{\tau_{i}} \in C^{0}\left(\psi_{i}\left(B_{i}\right)\right)^{N *}$, such that

$$
\mu(w)=\sum_{i=1}^{r} \mu_{i}\left(\Psi_{i} \circ w \mid B_{i} \circ \psi_{i}^{-1}\right) .
$$

Identifying $C^{0}\left(\psi_{i}\left(B_{i}\right)\right)^{N *}$ with $C^{0}\left(\psi_{i}\left(B_{i}\right)\right)^{* N}$ and observing that $C^{0}\left(\psi_{i}\left(B_{i}\right)\right)^{*}$ is the space of Radon measures on $\psi_{i}\left(B_{i}\right)$, we conclude that each $\mu_{i}$ is a collection of $N$ measures on $\psi_{i}\left(B_{i}\right)$.

Let $\left\{U_{\alpha}, \Psi_{\alpha}, \psi_{\alpha}\right\}$ be a vector bundle atlas of $\pi$, and let $C_{c}^{0}\left(\psi_{\alpha}\left(U_{\alpha}\right)\right)$ denote the Banach space of continuous functions with compact support in $\psi_{\alpha}\left(U_{\alpha}\right)$ equipped with the usual topology so that $C_{c}^{0}\left(\psi_{\alpha}\left(U_{\alpha}\right)\right)^{*}$ is the space of Radon measures on $\psi_{\alpha}\left(U_{\alpha}\right)$. Assume that for each $\alpha$ there is a given $\mu_{\alpha} \in C_{c}^{0}\left(\psi_{\alpha}\left(U_{\alpha}\right)\right)^{* N}$, such that for each pair of indices, $\tilde{\mu}_{\alpha}\left(\Psi_{\alpha} \circ w \circ \psi_{\alpha}^{-1}\right)=\mu_{\alpha}\left(\Psi_{\beta} \circ w \circ \psi_{\beta}^{-1}\right)$, for each $w \in C^{0}(\pi)$ whose support is contained in $U_{\alpha} \cap U_{\beta}$. We now define $\mu \in C^{0}(\pi)^{*}$ by

$$
\mu(w)=\sum_{i} \mu_{\sim}\left(\Psi_{i} \circ \phi_{i} w \circ \psi_{i}^{-1}\right), \quad w \in C^{0}(\pi),
$$

where $\left\{\phi_{i}\right\}$ is a finite partition of unity such that supp $\phi_{i} \subset U_{\alpha}$. It can be shown that $\mu$ is independent of the partition of unity so that any collection of local measures that satisfies the transformation rule define an element of $C^{0}(\pi)^{*}$.

Having reviewed the local properties of elements of $C^{0}(\pi)^{*}$, we extend them to a wider class of sections, the integrable sections. We say that a function $u_{i}: \quad \psi_{i}\left(U_{i}\right) \rightarrow R^{N}$ is integrable with respect to the collection of measures $\left\{\mu_{i k}\right\}, k=1, \ldots, N$, if each component is integrable with respect to all the $\mu_{i k}$, i.e., if

$$
\left\|u_{\sim}\right\|_{L^{\prime}}=\sup _{j, k} \int_{\psi_{i}\left(u_{i}\right)}\left|\underline{u}_{i j}\right| d\left|\mu_{\sim}\right|<\infty
$$


It can be shown that for a section $u$ of $\pi$ with support in $U_{i} \cap U_{j}$ and measures $\mu_{i}$ and $\mu_{j}$ on $\psi_{i}\left(U_{i}\right)$ and $\psi_{j}\left(U_{j}\right)$, respectively, satisfying the compatibility condition given above, $\Psi_{i} \circ u \circ \psi_{i}^{-1}$ is $\mu_{i}$ integrable if and only if $\Psi_{j} \circ u^{\circ} \psi_{j}^{-1}$ is $\mu_{j}$ integrable. Thus, we say that a section of $\pi$ is integrable with respect to $\mu \in C^{0}(\pi)^{*}$ if its local representatives are integrable with respect to the local representatives of $\mu_{i}$ of $\mu$.

Let $\chi_{T}$ be the characteristic function of a subset $T$ of $B$. If $T \cap U_{i}$ is $\mu_{i}$ measurable, $\chi_{T \cap U_{i}}$ is integrable with respect to each of the $\mu_{i k}, k=1, \ldots, N$, and we can restrict $\mu_{\sim} \boldsymbol{\mu}_{i}$ to $T \cap U_{i}$ by $\mu_{i} \mid T \cap U_{i}=\chi_{T \cap U_{i}} \mu_{i}$. In case the family $\left\{\mu_{\sim}\right\}$ satisfies the compatibility condition on the intersections of domains of charts so that it contains local representatives of some $\mu \in C^{0}(\pi)^{*}$, the same holds for the collection $\left\{\mu_{i} \mid T \cap U_{i}\right\}$ which will represent $\mu \mid T=\chi_{T} \mu$, the restriction of $\mu$ to $T$. In particular, if $P$ is a subbody of $B$, i.e., a compact $m$-dimensional submanifold of $B, \mu$ can be restricted to $P$.

Applying the foregoing results to the case where $\pi$ is the vector bundle $J^{p}\left(\kappa^{*}\left(\tau_{s}\right)\right)$, we conclude that a stress is represented locally by a collection of $N$ Radon measures that transform according to the rule given above, where $N$ is the dimension of the fiber of $J^{p}\left(\kappa^{*}\left(\tau_{s}\right)\right)$. Conversely, any such collection of measures satisfying the transformation rule represents a stress.

We denote the evaluation of the stress measure $\sigma \in C^{0}\left(J^{P}\left(\kappa^{*} \tau_{S}\right)\right)^{*}$ on a section $w$ by $\int_{B} w d \sigma$, and for a subbody $P$, we denote the evaluation of $\sigma \mid P$ on a section $u$ of $J^{P}\left((\kappa \mid P)^{*}\left(\tau_{S}\right)\right)$ by $\int_{P} u d \sigma$.

\section{THE CASE OF A CONNECTION}

We now assume that connections are specified both on the vector bundle $\tau_{S}: T S \rightarrow S$ and the vector bundle $\tau_{B}$ : $T B \rightarrow B$. The connection on $\tau_{S}$ induces a connection on $\kappa^{*} \tau_{B}$ and we recall that given a connection on both $\tau_{B}$ and $\kappa^{*} \tau_{B}$ we have an induced connection on the vector bundle of $p$-multilinear mappings $L^{p}\left(\tau_{B}, \kappa^{*} \tau_{S}\right): L^{p}\left(T B, \kappa^{*} T S\right) \rightarrow B$, such that we have covariant derivatives

$$
\nabla^{i} u \in C^{p-i}\left(L^{i}\left(\tau_{B}, \kappa^{*} \tau_{S}\right)\right), \quad 0 \leqslant i \leqslant p,
$$

for $u \in C^{p}\left(\kappa^{*} \tau_{s}\right)$ (see Eliasson ${ }^{11}$ for details).

Consider the mapping

$$
\underline{\nabla}^{p}: C^{p}\left(\kappa^{*} \tau_{S}\right) \rightarrow C^{0}\left(\underset{i=0}{\stackrel{p}{\oplus}} L^{i}\left(\tau_{B}, \kappa^{*} \tau_{S}\right)\right)
$$

given by

$$
u \mapsto\left(u, \nabla u, \ldots, \nabla^{p} u\right) .
$$

Again, this is a linear continuous injection with a closed image, and since

$$
\begin{aligned}
C^{0}\left(\underset{i=0}{\oplus} L^{i}\left(\tau_{B}, \kappa^{*} \tau_{S}\right)\right)^{*} & =\left(\underset{i=0}{\stackrel{p}{\oplus}} C^{0}\left(L^{i}\left(\tau_{B}, \kappa^{*} \tau_{S}\right)\right)\right)^{*} \\
& =\underset{i=0}{p} C^{0}\left(L^{i}\left(\tau_{B}, \kappa^{*} \tau_{S}\right)\right)^{*},
\end{aligned}
$$

we have a representation of forces by collections of tensor measures $\left(\sigma_{0}, \sigma_{1}, \ldots, \sigma_{p}\right), \sigma_{i} \in C^{0}\left(L^{i}\left(\tau_{B}, \kappa^{*} \tau_{S}\right)\right)^{*}$, in the form

$$
f(u)=\sum_{i=0}^{p} \int_{B} \nabla^{i} u d \sigma_{i}
$$

Thus, in case connections are specified on $\tau_{B}$ and $\tau_{S}$, forces can be represented by tensor valued measures. We note that the case $p=1$ corresponds to classical continuum mechanics, where $\sigma_{1}$ is the tensor measure which corresponds to the stress tensor. In this case we do not need the connection on $\tau_{B}$, as it is not required for the first covariant derivative.

\section{THE RELATION TO PREVIOUS WORKS}

In this section we review some ideas suggested in previous works, ${ }^{18-20}$ and relate them to the formulation given here.

In Refs. 19 and 20 it was suggested that vector bundles over $B$ and $S$ can serve as mathematical models for the local properties of both body and space so that the vector space attached to each point represents mathematically the neighborhood of this point. A local configuration was defined as a vector bundle morphism between the two vector bundles. The local configuration space, local virtual displacements, and local forces were defined for this new model, termed the local model, in analogy with the previous set of definitions which will be referred to henceforth as the global model. The local configuration space is the Banach manifold of all local configurations, local virtual displacements are elements of the tangent bundle, and local forces are elements of the cotangent bundle of the local configuration space. It was shown that local forces generalize the stresses of continuum mechanics, and the principle of virtual work was obtained as a result of a requirement for compatibility between these two models. The particular case where $\tau_{B}$ and $\tau_{S}$ represented the body and space in the local model was studied. In this case the local configuration space is the collection of vector bundle morphisms $\tau_{B} \rightarrow \tau_{S}$ which can be identified with the collection of sections of the jet bundle $\pi^{1}: J^{1}(B, S) \rightarrow B$.

Using the language of jet bundles and the properties of manifolds of sections of jet bundles, ${ }^{10}$ the following obvious generalization can be made. A local configuration of order $p$ is a continuous section of $\pi^{P}: J^{P}(B, S) \rightarrow B$. The local configuration space is the manifold of sections $C^{0}\left(\pi^{P}\right)$. A local virtual displacement is an element of the tangent bundle $T C^{0}\left(\pi^{p}\right)$, and a local force $\sigma$ is an element of the cotangent bundle $T^{*} C^{0}\left(\pi^{P}\right)$.

Since both the global model and the local model represent the same physical phenomenon they are related by compatibility conditions in the following way.

Consider the jet extension mapping

$$
j_{p}: \quad C^{p}\left(\pi^{0}\right) \rightarrow C^{0}\left(\pi^{p}\right) .
$$

We say that a local configuration $\chi \in C^{0}\left(\pi^{P}\right)$ is compatible with a global configuration $\kappa \in C^{p}\left(\pi^{0}\right)$ if $\chi=j_{p}(\kappa)$. A local virtual displacement $w \in T C^{0}\left(\pi^{p}\right)$ is compatible with a global virtual displacement $u \in T C^{p}\left(\pi^{0}\right)$ if $w=T\left(j_{p}\right)(u)$. We say that a global force $f \in T^{*} C^{P}\left(\pi^{0}\right)$ is compatible with the local force $\sigma \in T^{*} C^{0}\left(\pi^{P}\right)_{\mid \text {image } j_{p}}$ if $f=T^{*}\left(j_{p}\right)(\sigma)$. These definitions can be summarized by saying that the two models are related by the jet functor $J^{P}$.

The relation between the formulation given in this section and the rest of this paper is established in the following proposition. 
Proposition 6.1: (i) For any global configuration, $\kappa, T^{*} C^{0}\left(\pi^{p}\right)_{j_{p}(\kappa)}$, the space of local forces at the local configuration compatible with $\kappa$, can be identified with the space of stresses representing forces at $\kappa$.

(ii) A global force $f$ is compatible with a local force $\sigma$ if and only if the stress that can be identified with $\sigma$ by (i) represents $f$.

Proof: The proof of the proposition becomes obvious once the following results of Palais ${ }^{10}$ on sections of jet bundles are used.

(a) Given $\kappa \in C^{p}\left(\pi^{0}\right)$, there is a natural isomorphism $T C^{0}\left(\pi^{p}\right)_{j(\alpha)} \cong C^{0}\left(J^{P}\left(\kappa^{*} \tau_{S}\right)\right)$.

(b) For $\kappa \in C^{P}\left(\pi^{0}\right)$, the tangent to the jet extension mapping

$$
T\left(j_{p}\right)_{\kappa}: T C^{p}\left(\pi^{0}\right)_{\kappa} \rightarrow T C^{0}\left(\pi^{p}\right)_{j_{p}(\kappa)}
$$

is given by $u \mapsto j_{p}(u)$, where $u \in C^{p}\left(\kappa^{*} \tau_{S}\right) \cong T C^{p}\left(\pi^{0}\right)_{\kappa}$ and the identification of (i) is used.

The assertions follow immediately.

\section{FORCE SYSTEMS}

The representation of forces by stress measures provides an answer to the basic problem of restriction of forces to subbodies. Given a stress measure $\sigma$, a unique force $f_{P}$ is induced on every subbody $P$ by

$$
f_{P}(u)=\int_{P} j_{p}(u) d \sigma, \quad u \in C^{P}\left((\kappa \mid P)^{*} \tau_{s}\right)
$$

or in other words, the force on $P$ is represented by the restriction of the stress measure to $P$.

We will use the term force system for a set function assigning a force $f_{P} \in C^{P}\left((\kappa \mid P)^{*} \tau_{S}\right)^{*}$ to every subbody $P$ of $B$. We will say that a force system is consistent if there exists a stress representation $\sigma$ such that the force given on any subbody $P$ is represented by the restriction of $\sigma$ to $P$.

Since the jet extension map is not surjective we cannot expect that the representation of forces by stresses will be unique. This feature is well known in continuum mechanics and it is referred to as static indeterminacy. It is the static indeterminacy which forces the use of material properties or constitutive relations in order to be able to restrict forces to subbodies. However, as the next proposition shows, a force system can be consistent with at most one stress, i.e., if we know the force acting on each subbody we can determine the stress uniquely. This statement is a generalization of the principle in continuum mechanics according to which the stress at a point can be determined uniquely if the traction across every surface is given. In the classical case however, the result is stated for the case $p=1$ only, and the stress tensor measure is given in terms of a tensor field whose value at a given point we want to determine.

Proposition 7.1: If a force system is consistent with the stresses $\sigma_{1}$ and $\sigma_{2}$ then, $\sigma_{1}=\sigma_{2}$.

Proof: In order to show that $\sigma_{1}=\sigma_{2}$, it suffices to show that their local representatives in any given chart are equal. Let $\boldsymbol{A}$ be a subbody contained in the domain of a chart in $B$. For any subbody $P$ of $A$, let $f_{P}$ be the force acting on $P$ in the given force system which is consistent with both $\sigma_{1}$ and $\sigma_{2}$.
We denote by $u^{i}$ the components of the local representative of $u \in C^{p}\left((\kappa \mid P)^{*} \tau_{s}\right)$ and for the multi-index $\alpha=\left(\alpha_{1}, \ldots, \alpha_{m}\right)$, we recall that the local representative of $j_{p}(u)$ is $\left\{D^{\alpha} u^{i}\right\},|\alpha|=\alpha_{1}+\cdots+\alpha_{m} \leqslant p$. Let $\mu_{i \alpha}$ and $v_{i \alpha}$ be the measures on $P$-the image of $P$ under the chart-that represent $\sigma_{1}$ and $\tilde{\sigma}_{2}$, respectively. By the representation of forces by stresses we have

$$
f_{P}(u)=\int_{\underline{P}|\alpha|=0} \sum^{p} D^{\alpha} u^{i} d \mu_{i \alpha}=\int_{\underline{P}|\alpha|=0} \sum^{p} D^{\alpha} u^{i} d v_{i \alpha}
$$

for every $u \in C^{P}\left((\kappa \mid P)^{*} \tau_{S}\right)$ and every subbody $P$.

In particular, for $j \in\{1, \ldots, n\}$, where $n$ is the dimension of $S$, let $u$ satisfy $u^{i}=\delta^{i j}$. By the equation above we have

$$
\mu_{j \alpha}(\underset{\sim}{P})=v_{j \alpha}(\underset{\sim}{P}), \quad|\alpha|=0,
$$

for every subbody $P$ of $A$. Since the two measures agree on every subbody we have $\mu_{j \alpha}=v_{j \alpha},|\alpha|=0$.

Now, given $j, \beta$, with $|\beta|=1$, let $u$ satisfy $u^{i}=\delta^{i j} x^{\beta}$, where $\left(x^{k}\right)$ are the local coordinates in the given chart. We have

$$
\int_{P} \sum_{|\alpha|<1} D^{\alpha} u^{i} d \mu_{i \alpha}=\int_{P} \sum_{|\alpha|<1} D^{\alpha} u^{i} d v_{i \alpha}
$$

as the higher-order derivatives vanish. Since $\mu_{i \alpha}=v_{i \alpha}$ for $|\alpha|=0$, and since $D^{\alpha} u^{i} \neq 0$ only for $i=j, \alpha=\beta$, we have $\mu_{j \beta}(\underset{\sim}{P})=v_{j \beta}(\underset{\sim}{P})$, for every subbody $P$ and arbitrary $j, \beta$, $|\beta|=1$. We conclude that that $\mu_{i \alpha}=v_{i \alpha}$ for all $i$ and $\alpha$, with $|\alpha|=1$.

We can continue the process evaluating the virtual work performed on the virtual displacements $u$ such that $u^{i}=\delta^{i j} x^{\alpha}=\delta^{i j}\left(x^{1}\right)^{\alpha_{1}}\left(x^{2}\right)^{\alpha_{2}} \ldots\left(x^{n}\right)^{\alpha_{n}}$, with $|\alpha|=2,3, \ldots, p$ to obtain $\mu_{i \alpha}=v_{i \alpha}$ for all $\alpha$ with $|\alpha| \leqslant p$.

Proposition 7.2: Let a force system $\left\{f_{P}\right\}$ which is consistent with a stress $\sigma$, be given. Then, if $A$ is a subbody contained in the domain of a chart on $\kappa^{*} \tau_{S}$ with coordinates $\left(x^{k}, u^{j}\right)$, the local representatives $\mu_{i \alpha}$ of $\sigma$ are given by the following inductive process.

Let $\left(\delta^{i j} x^{\alpha}\right)^{\prime}$ be the section of $C^{p}\left((\kappa \mid A)^{*} \tau_{S}\right)$ whose local representatives satisfy $u^{i}=\delta^{i j} x^{\alpha}$ for given $j$ and $\alpha$. Then,

$$
\begin{aligned}
\mu_{j \alpha}(\underset{\sim}{P}) & =f_{P}\left(\delta^{i j}\right)^{\prime}, \quad|\alpha|=0 \\
\mu_{j \alpha}(\underset{\sim}{P})= & \frac{1}{\alpha !} f_{P}\left(\delta^{i j} x^{\alpha}\right)^{\prime} \\
& \quad-\sum_{\beta<\alpha} \frac{1}{(\alpha-\beta) !} \int_{P} x^{\alpha-\beta} d \mu_{j \beta}, \\
0<|\alpha| \leqslant p &
\end{aligned}
$$

where $\beta<\alpha$ means that $\beta_{i} \leqslant \alpha_{i}$ and $|\beta|<|\alpha|$.

Proof: By hypothesis

Since,

$$
f_{p}\left(\delta^{i j} x^{\alpha}\right)^{\prime}=\int_{\underline{P}|\beta|<p} \sum_{\beta} D^{\beta}\left(\delta^{i j} x^{\alpha}\right) d \mu_{i \beta}
$$

$$
D^{\beta} x^{\alpha}=[\alpha ! /(\alpha-\beta) !] x^{\alpha-\beta}
$$

for $\beta<\alpha$, and $D^{\beta} x^{\alpha}=0$, for $\alpha<\beta$, we have 


$$
\begin{aligned}
f_{P}\left(\delta^{i j} x^{\alpha}\right)^{\prime}= & \delta^{i j} \alpha ! \mu_{i \alpha}(\underset{\sim}{P}) \\
& +\sum_{\substack{\beta \neq \alpha \\
|\beta|<p}} \frac{\alpha !}{(\alpha-\beta) !} \int_{P} \delta^{i j^{\alpha}} x^{\alpha-\beta} d \mu_{i \beta} \\
= & \alpha ! \mu_{j \alpha}(\stackrel{P}{P}) \\
& +\sum_{\beta<\alpha} \frac{\alpha !}{(\alpha-\beta) !} \int_{P} x^{\alpha-\beta} d \mu_{j \beta} .
\end{aligned}
$$

The proposition suggests a procedure which enables one to determine whether a given force system is consistent with any stress, and to obtain the local representatives of this stress if it exists. Given any vector bundle atlas on $\kappa^{*} \tau_{S}$, one has to evaluate $f_{P}\left(\delta^{i j}\right)^{\prime}$ for all subbodies $P$ contained in the domain of charts. Then, if for every chart, the set function $P \mapsto f_{P}\left(\delta^{i j}\right)^{\prime}$ for all subbodies contained in the domain of the chart can be extended to a measure on the domain of the chart, we can identify $\mu_{j \alpha}(\underset{\sim}{P}),|\alpha|=0$, with $f_{P}\left(\delta^{i j}\right)^{\prime}$ for the various charts. We proceed by evaluating $f_{P}\left(\delta^{i j} x^{\alpha}\right)^{\prime},|\alpha|=1$, and we use the relations of the last proposition and the previously obtained $\mu_{j \alpha}(\underset{\sim}{P}),|\alpha|=0$, to obtain $\mu_{j \alpha}(P),|\alpha|=1$. We check that $\mu_{j \alpha},|\alpha|=1$, can be extended to measures and we continue the process for $f_{P}\left(\delta^{i j} x^{\alpha}\right)^{\prime},|\alpha|>1$, until we reach a $p$ th step such that $\mu_{j \alpha}=0$ for all $|\alpha|>p$. Next, we have to check that the $\mu_{j \alpha}$ satisfy the transformation rules on the intersections of charts. If the compatibility conditions are satisfied, we conclude that the $\left\{\mu_{j \alpha}\right\},|\alpha|<p$, obtained are the local representatives of a stress which is consistent with the given force system.

\section{CONSTITUTIVE RELATIONS}

As we mentioned in the introduction, the problem of the restriction of forces to subbodies, which was transformed into a problem of nonunique relation between forces and stresses, leads to the specification of the material properties as additional information. The material properties are introduced via the so called constitutive relations, which in classical continuum mechanics, associate the stress at a point with the deformation gradient at that point. In this section we suggest a way by which constitutive theory may be incorporated in the structure that we developed.

We assume that the following two principles hold in continuum mechanics.

Axiom 8.1. (the principle of body self-determinism): The force acting on a body is determined by the configuration of the body, i.e., for any body $B$ there is a section $F_{B}: Q \rightarrow T^{*} Q$ which we call the loading of $B$.

Axiom 8.2 (the principle of consistency): Given any configuration $\kappa$ of the body $B$, the force system $\left\{F_{P}(\kappa \mid P) ; P\right.$ is a subbody of $B$ \}, is consistent.

Thus, by Proposition 7.1, the principle of consistency implies that any configuration of $B$ determines a unique stress representation in $T^{*} C^{0}\left(\pi^{P}\right)$. The mapping $\Psi_{B}: Q \rightarrow T^{*} C^{0}\left(\pi^{p}\right)$ that associates stresses with the various configurations is called a constitutive relation for $B$.
Given a force $f \in T^{*} Q$ and a constitutive relation $\Psi_{B}$ such that $f=T^{*}\left(j_{p}\right)\left(\Psi_{B}(\kappa)\right)$, the measure $\Psi_{B}(\kappa)$ induces a unique force on any subbody and the problem of the restriction of the force is immediately solved. The general problem of continuum mechanics can be formulated now as follows. Given a loading $F_{B}$ of $B$ and a constitutive relation $\Psi_{B}$, determine the configuration $\kappa$ such that $\Psi_{B}(\kappa)$ represents $F_{B}(\kappa)$, i.e., $F_{B}(\kappa)=T^{*}\left(j_{p}\right)\left(\Psi_{B}(\kappa)\right)$.

It should be noted that in the general geometric framework we use, any "force" is a "follower force" in the sense that a force has meaning only when it is associated with a configuration. Thus, rather than looking for an equilibrium configuration under a given force, a meaningless problem, one has to find the equilibrium configuration for a given loading.

We can examine now the way in which the principle of local determinism restricts the constitutive relations. Let $P$ be subbody of $B$ and let $\Psi_{P}, \Psi_{B}$ be constitutive relations on $P$ and $B$, respectively. Since for any $\kappa$, the principle of body determinism implies that the force on $P$ and any of its subbodies is determined by $\kappa \mid P$, we have $\Psi_{P}(\kappa \mid P)=\Psi_{B}(\kappa) \mid P$. Thus, we will omit the suffix and we will write $\Psi$ when no confusion can arise. We also note that this principle implies that it is sufficient to examine the case where $B$ is in $R^{m}$. Moreover, assuming that the constitutive relations are continuous, we can show that the constitutive relations are $p$-jet local in the following sense.

Proposition 8.3: Let $\Psi$ be a continuous constitutive relation and let $x \in B$. Then, for any $\epsilon>0$, there exists a $\delta>0$ such that if a subbody $P$ is contained in a ball of radius $\delta$ (in the $R^{m}$ Euclidean metric) centered at $x$, then,

$$
\left\|\Psi(\kappa)\left|P-\Psi\left(j_{p}(\kappa)(x)\right)\right| P\right\|<\epsilon,
$$

where $j_{p}(\kappa)(x)$ denotes the $p$ th-order Taylor expansion of $\kappa$ about $x$.

Proof: Given any $\epsilon>0$, the continuity of $\Psi$ implies that there exists a $\delta_{1}>0$ such that if $\left\|\kappa-j_{p}(\kappa)(x)\right\|_{C^{p}}<\delta_{1}$, then $\left\|\Psi(\kappa)-\Psi\left(j_{p}(\kappa)(x)\right)\right\|<\epsilon$. By Taylor's theorem, given $\delta_{1}>0$, there is a $\delta>0$ such that $\left\|\kappa\left|P-j_{p}(\kappa)(x)\right| P\right\|_{C^{p}}<\delta_{1}$ if $P$ is contained in a ball of radius $\delta$ about $x$. Thus, by locality

$$
\begin{aligned}
& \left\|\Psi(\kappa)\left|P-\Psi\left(j_{p}(\kappa)(x)\right)\right| P\right\| \\
& \quad=\left\|\Psi(\kappa \mid P)-\Psi\left(j_{p}(\kappa)(x) \mid P\right)\right\|<\epsilon .
\end{aligned}
$$

Since there is no meaning to the value of a stress at a point, the classical locality assumption that the value of the stress at a point depends on the value of the deformation gradient at that point cannot be obtained or even conjectured. If stresses were continuous sections and if the space of stresses were given the $C^{1}$ topology, then the continuity argument of the previous proposition together with the two principles would imply that the value of the stress at a point depends only on the value of the $p$ th jet at that point.

\section{STRESSES GIVEN BY SNOOTH DENSITIES}

In this section, in order to complete the analogy with classical continuum mechanics, we obtain the representation of forces by surface forces and body forces, the equilibrium differential equations, and the boundary conditions. 
Since the procedure involves integration by parts, we assume that the stresses are given in terms of smooth densities. We also assume that a connection is specified on $S$ and that $B$ and $S$ have the same dimension. It follows that the connection on $S$ induces a connection on $B$. Keeping $\kappa$ fixed during the discussion, we identify the body with its image under $\kappa$.

We saw that if connections are given on $B$ and $S$, any force can be represented in the form

$$
f(u)=\sum_{k=0}^{p} \int_{B} \nabla^{k} u d \sigma_{k},
$$

where $\sigma_{k}$ is the $k$ th-order stress measure. Consider the vector bundle $L\left(L^{k}\left(\tau_{B}, \kappa^{*} \tau_{S}\right), \stackrel{m}{\wedge} T^{*} B\right)$. Assuming that $B$ is orientable, a smooth section $s_{k}$ of this vector bundle induces a $k$ th-order stress measure $\sigma_{k}$ by

$$
\int_{B} \nabla^{k} u d \sigma_{k}=\int_{B} s_{k} \circ \nabla^{k} u,
$$

where $s_{k} \circ \nabla^{k} u$ is the $m$-form whose value at $x \in B$ is $s_{k}(x)\left(\nabla^{k} u(x)\right)$. In particular, if a volume element $\theta$ is given on $B$, the collection of sections $\left\{s_{k}\right\}, s_{k} \in C^{\infty}\left(L^{k}\left(\tau_{B}, \kappa^{*} \tau_{S}\right)^{*}\right)$ will induce a stress representation

$$
f(u)=\sum_{k=0}^{p} \int_{B} s_{k}\left(\nabla^{k} u\right) \theta
$$

where $s_{k}\left(\nabla^{k} u\right)$ is the real function whose value at $x \in B$ is $s_{k}(x)\left(\nabla^{k} u(x)\right)$. More geometric structure is available in the case where both the connection and the volume element are derived from a Riemmanian metric.

In order to perform the integration by parts in the general geometric framework, we generalize the definition of the divergence of a tensor field as follows. We have the isomorphism

$$
\begin{aligned}
& L\left(L^{k}\left(\tau_{B}, \kappa^{*} \tau_{S}\right), \stackrel{m}{\wedge} T^{*} B\right) \\
& \quad \cong \stackrel{m}{\wedge} T^{*} B \otimes \tau_{B} \otimes L^{k-1}\left(\tau_{B}, \kappa^{*} \tau_{S}\right)^{*}
\end{aligned}
$$

and we define

$$
\text { co: } L\left(L^{k}\left(\tau_{B}, \kappa^{*} \tau_{S}\right), \stackrel{m}{\wedge} T^{*} B\right)
$$

$$
\rightarrow L\left(L^{k-1}\left(\tau_{B}, \kappa^{*} \tau_{S}\right), \wedge^{m-1} T^{*} B\right),
$$

to be the mapping induced by the contraction of the first two factors in the tensor product above. Then, for $s_{k} \in C^{\infty}\left(L\left(L^{k}\left(\tau_{B}, \kappa^{*} \tau_{S}\right), \stackrel{m}{\wedge} T^{*} B\right)\right)$, we define the divergence $\operatorname{div} s_{k} \in C^{\infty}\left(L\left(L^{k-1}\left(\tau_{B}, \kappa^{*} \tau_{S}\right), \stackrel{m}{\wedge} T^{*} B\right)\right)$ by

$$
\operatorname{div} s_{k}\left(\nabla^{k-1} u\right)=d\left(\operatorname{co}\left(s_{k}\left(\nabla^{k-1} u\right)\right)\right)-s_{k}\left(\nabla^{k} u\right) .
$$

Using local expressions it can be shown that the divergence is well defined and that it agrees with the usual definition in the case of a Riemannian manifold.

For the case $p=1$, let the force $f$ be represented by smooth densities in the form

$$
f(u)=\int_{B} s_{0}(u)+s_{1}(\nabla u),
$$

where $s_{i} \in C^{\infty}\left(L\left(L^{i}\left(\tau_{B}, \kappa^{*} \tau_{S}\right), \stackrel{m}{\wedge} T^{*} B\right)\right)$. Using the definition of the divergence and Stokes' theorem, one can show that $f$ can be represented by two sections $b \in C^{\infty}\left(L\left(\tau_{B}, \stackrel{m}{\wedge} T^{*} B\right)\right)$ and $t \in C^{\infty}\left(L\left(i^{*} \tau_{B}, \stackrel{m-1}{\wedge} T^{*} \partial B\right)\right)(i$ is the embedding $\partial B \rightarrow B$ ) in the form

$$
f(u)=\int_{B} b(u)+\int_{\partial B} t(u),
$$

where $b$ and $t$ satisfy $\operatorname{div} s_{1}+b=s_{0}$ and $t=i^{*} \circ \operatorname{co}\left(s_{1}\right)$. (We use $i^{*}$ for both the pullback of differential forms and the pullback of vector bundles.)

In the case of Riemannian geometry we obtain for the three-dimensional case the usual result, i.e., if

$$
f(u)=\int_{B}\left(s_{0 j} u^{j}+s_{1 j}^{i} u^{j}{ }_{i}\right) d v
$$

(the vertical bar denotes covariant derivative), we have

$$
f(u)=\int_{B} b_{j} u^{j} d v+\int_{\partial B} t_{j} u^{j} d a,
$$

where $s_{1 j \mid i}^{i}+b_{j}=b_{0 j}, \quad t_{j}=n_{i} s_{1 j}^{i}$, and $n$ is the unit normal to the boundary.

Remark: The term $s_{0}$ vanishes and the term $s_{1}$ can be shown to be symmetric in the Euclidean geometry if we require that the force is invariant with respect to the Euclidean group (cf. Refs. 4, 21, and 22).

For the case $p=2$, the case of second-grade continuum mechanics, we assume that the stresses are given in terms of the densities $s_{0}, s_{1}$, and $s_{2}$ such that

$$
f(u)=\int_{B}\left(s_{0}(u)+s_{1}(\nabla u)+s_{2}\left(\nabla^{2} u\right)\right)
$$

It can be shown that $f$ can be represented in the form

$$
f(u)=\int_{B} b(u)+\int_{\partial B}\left(t(u)+t^{\prime}(\nabla u)\right),
$$

where $b, t$, and $t^{\prime}$ are in

$$
C^{\infty}\left(L\left(\tau_{B}, \stackrel{m}{\wedge} T^{*} B\right)\right), \quad C^{\infty}\left(L\left(i^{*} \tau_{B}, \stackrel{m-1}{\wedge} T^{*} \partial B\right)\right),
$$

and

$$
C^{\infty}\left(L\left(\mathrm{~L}\left(i^{*} \tau_{B}, i^{*}\left(\kappa^{*} \tau_{S}\right)\right), \stackrel{m-1}{\wedge} T^{*} \partial B\right)\right),
$$

respectively, and they satisfy

$$
\begin{aligned}
& b=\operatorname{div}^{2} s_{2}-\operatorname{div} s_{1}+s_{0}, \\
& t=i^{*} \circ \operatorname{co}\left(s_{1}\right)-i^{* \circ} \operatorname{co}\left(\operatorname{div} s_{2}\right), \\
& t^{\prime}=i^{*} \circ \operatorname{co}\left(s_{2}\right) .
\end{aligned}
$$

A further integration by parts of the term involving $t$ ' is possible only if we have additional geometric structure. Again, for the three-dimensional Riemannian geometry, the classical results (see, e.g., Refs. 23 and 24) can be obtained.

'M. E. Gurtin and W. O. Williams, "An axiomatic foundation for continuum thermodynamics," Arch. Ration. Mech. Anal. 26, 83 (1967).

${ }^{2}$ M. E. Gurtin and L. C. Martins, "Cauchy's theorem in classical physics," Arch. Ration. Mech. Anal. 60, 303 (1976). 
${ }^{3}$ C. Truesdell, A First Course in Rational Continuum Mechanics (Academic, New York, 1977), Vol. I.

${ }^{4} \mathrm{~J}$. Marsden and T. Hughes, Mathematical Foundations of Elasticity (Prentice-Hall, Englewood Cliffs, NJ, 1983).

${ }^{5}$ C. Truesdell and W. Noll, "The nonlinear field theories of mechanics," in Handbuch der Physik, edited by S. Flugge (Springer, Berlin, 1965), Vol. III $/ 3$.

${ }^{6}$ R. D. Mindlin, "Elasticity, piezoelectricity, and crystal lattice dynamics," J. Elasticity, 2, 217 (1972).

${ }^{7}$ V. I. Amold, Mathematical Methods of Classical Mechanics (Springer, New York, 1978).

${ }^{8} \mathrm{~W}$. Tulczyjew, "Simple models of Lagrangian submanifolds of symplectic manifolds," Rend. Sem. Mat. Univ. Politec. Torino 35, 97 (1977).

${ }^{9} \mathrm{C}$. Truesdell and R. Toupin, "The classical field theories," in Handbuch der Physik, edited by S. Flugge (Springer, Berlin, 1960), Vol. III/1.

${ }^{10} \mathrm{R}$. Palais, Foundations of Global Non-Linear Analysis (Benjamin, New York, 1968).

"I. E. Eliasson, "Geometry of manifolds of maps," J. Differential Geom. 1, 169 (1967).

${ }^{12}$ P. W. Michor, Manifolds of Differentiable Mappings (Shiva, London, 1980).

${ }^{13} \mathrm{~N}$. Krikorian, "Differentiable structures on function spaces," Trans. Am. Math. Soc. 171, 67 (1972).
${ }^{14}$ M. Golubitsky and V. Guillemin, Stable Moppings and Their Singularities (Springer, New York, 1973).

${ }^{15}$ Y. Choquet-Bruhat, C. DeWitt-Morette, and M. Dillard-Bleick, Analysis, Manifolds, and Physics (North-Holland, Amsterdam, 1977).

${ }^{16} \mathrm{G}$. de Rham, Varietes Differentiables (Hermann, Paris, 1955).

${ }^{17} \mathrm{~L}$. Schwartz, Theorie des Distributions (Hermann, Paris, 1966),

${ }^{18} M$. Epstein and R. Segev, "Differentiable manifolds and the principle of virtual work in continuum mechanics," J. Math. Phys. 21, 1243 (1980).

${ }^{19} \mathrm{R}$. Segev, "Differential Manifolds and Some Basic Notions of Continuum Mechanics," Ph.D. thesis, Dept. Mech. Eng., Univ. of Calgary, 1981.

${ }^{20}$ R. Segev and M. Epstein, "An invariant theory of stress and equilibrium," in Mathematical Foundations of Elasticity, edited by J. Marsden and T. Hughes (Prentice-Hall, Englewood Cliffs, 1983), pp. 169-175.

${ }^{21} \mathrm{~W}$. Noll, "La mecanique classique basee sur un axiome d'objectivite," in La Methode Axiomatique dans les Mecaniques Classiques et Nouvelles (Gauthier-Villars, Paris, 1963).

${ }^{22}$ A. M. Green and R. S. Rivlin, "On Cauchy's equations of motion," J. Appl. Math. Phys. 15, 290(1964).

${ }^{23}$ R. Toupin, "Elastic materials with couple stresses," Arch. Ration. Mech. Anal. 11, 385 (1962).

${ }^{24}$ R. D. Mindlin, "Micro structure in linear elasticity," Arch. Ration. Mech. Anal. 16, 51 (1964). 